\section{Lungengesundheit: Beruf als großer Risikofaktor?}

Blanc PD et al. The Occupational Burden of Nonmalignant Respiratory Diseases. An Official American Thoracic Society and European Respiratory Society Statement. Am J Respir Crit Care Med 2019; doi:10.1164/rccm.201904-0717ST

Die berufsbedingte Exposition gegenüber Noxen wie Quarzstaub oder Asbest ist als relevanter Risikofaktor bei der Pathogenese nicht-maligner Lungenerkrankungen anerkannt. Welche negativen Effekte die Belastung durch den Beruf und das Arbeitsleben allgemein für die Lungengesundheit haben kann, ist bis heute unklar. Blanc und Kollegen haben daher eine umfassende Recherche durchgeführt und kommen in ihren Schätzungen zu einem alarmierenden Ergebnis.

Zur effektiven Prävention, Früherkennung und Therapie von Lungenerkrankungen sind Kenntnisse über potenzielle Risikofaktoren besonders wichtig. Zudem spielt der prozentuale Beitrag von berufsbedingten Expositionsfaktoren im Rahmen der Pathogenese von Krankheitsbildern wie Asthma bronchiale über die Lungenfibrose bis hin zu Atemwegsinfektionen einschließlich Pneumonie und Tuberkulose eine große Rolle.

Um die Bedeutung des Arbeitsplatzes und des Berufes allgemein für die Entstehung dieser und weiterer pneumologischer Krankheitsbilder einmal genau beziffern zu können, haben Blanc und seine Arbeitsgruppe im Namen der American Thoracic Society eine umfassende Literaturrecherche zum Thema durchgeführt und ein entsprechendes Statement veröffentlicht. Sie recherchierten dafür in den internationalen Datenbanken PubMed und Embase nach Studien, die eine grobe Einschätzung des prozentualen Beitrages berufsbedingter Faktoren für ausgewählte Lungenerkrankungen möglich machten.
Sie differenzierten auf Ebene der Krankheitsbilder 4 Kategorien:

- Atemwegserkrankungen wie Asthma bronchiale, COPD und chronische Bronchitis.

- Interstitielle Lungenerkrankungen wie idiopathische Lungenfibrose.

- Granulomatöse Prozesse wie Sarkoidose.

- Atemwegsinfektion wie Tuberkulose oder Pneumonie.

Für jedes Krankheitsbild nahmen sie dann insbesondere Fall-Kontroll-Studien genauer unter die Lupe und errechneten bzw. schätzten den Anteil, den die Exposition gegenüber bestimmter Noxe am Arbeitsplatz bei der Krankheitsentstehung beigetragen haben könnte. Die übergeordnete Zielsetzung der Arbeitsgruppe lag darin, für jede der ausgewählten Atemwegserkrankungen den Berufsbeitrag zur Belastung möglichst genau beziffern zu können.

\section{Beruf als Belastungsfaktor}

Auf Basis der Ergebnisse der Literaturrecherche konnten die Studienautoren zunächst feststellen, dass die Exposition am Arbeitsplatz erheblich zur Belastung der Gesamtbevölkerung durch multiple chronische Atemwegserkrankungen beiträgt. So schätzen sie den Einfluss des Arbeitsplatzes bei der Pathogenese von Asthma bronchiale auf $16 \%$, für die chronisch obstruktive Lungenerkrankung und chronische Bronchitis konnten sie den Einfluss auf $14 \%$ bzw. 13\% beziffern. Entsprechende Zahlen für die idiopathische Lungenfibrose sowie eine hypersensitive Pneumonitis lagen sogar bei $26 \%$ und $19 \%$. In der Gruppe der granulomatösen Erkrankungen lag der Beitrag durch die berufliche Belastung beim Krankheitsbild der Sarkoidose bei 30\% und bei der Lungenalveolarproteinose bei $29 \%$.

Für Lungeninfektionen wie Tuberkulose und die ambulant erworbene Pneumonie bei Erwachsenen im erwerbsfähigen Alter konnten die entsprechenden Zahlen auf $2,3 \%$ und $10 \%$ beziffert werden. Auf Basis dieser Resultate ziehen die Studienautoren das Fazit, dass der Beruf einen erheblichen und auf Basis von Studiendaten nachweisbaren Beitrag bei der Pathogenese der hier vorgestellten Lungenerkrankungen leisten würde. Sie fordern daher sowohl auf klinischer, Forschungsund vor allem auf gesundheitspolitischer Ebene Konsequenzen.

\section{FAZIT}

Die Ergebnisse dieser umfassenden Übersichtsarbeit machen deutlich, dass die berufsbedinge Exposition gegenüber Noxen eine prozentual große Rolle bei der Pathogenese von nichtmalignen Lungenerkrankungen spielt. So konnten die Studienautoren den Beitrag des Faktors Arbeitsplatz für Asthma, Sarkoidose und Tuberkulose auf $16 \%, 30 \%$ und $2,3 \%$ schätzen. Sie stufen diese Zahlen als alarmierend ein und wünschen sich mehr Aufmerksamkeit im klinischen Bereich und auf gesundheitspolitischer Ebene.

Dipl.-Psych. Annika Simon, Hannover

Hinweis: Dieser Beitrag wurde gemäß Erratum vom 17.10.2019 geändert. 